\title{
A gas-rich disk around DX Cha
}

\author{
A. S. Hales ${ }^{1,2}$, I. De Gregorio-Monsalvo ${ }^{1,3}$, B. Montesinos ${ }^{4}$, \\ S. Casassus, W. R. F. Dent, C. Eiroa, A. M. Hughes, G. Garay, \\ D. Mardones, F. Ménard, A. Palau, S. Pérez, N. Phillips, \\ J. M. Torrelles and D. Wilner \\ ${ }^{1}$ Atacama Large Millimeter/Submillimeter Array, Joint ALMA Observatory, Alonso de \\ Córdova 3107, Vitacura 763-0355, Santiago - Chile \\ email: ahales@alma.cl \\ ${ }^{2}$ National Radio Astronomy Observatory, 520 Edgemont Road, Charlottesville, Virginia, \\ 22903-2475, United States \\ ${ }^{3}$ European Southern Observatory, Karl-Schwarzschild-Str. 2, 85748, Garching bei Mnchen, \\ Germany \\ ${ }^{4}$ Department of Astrophysics, Centre for Astrobiology (CAB, CSIC-INTA), ESAC Campus, \\ P.O. Box 78, 28691 Villanueva de la Cañada, Madrid, Spain
}

\begin{abstract}
DX Cha (HD 104237) is a southern, optically bright Herbig Ae star with an infrared excess, that is part of a small stellar group younger than 5 Myr. We used the APEX and ASTE submillimeter telescopes in Chile to search for continuum and gas emission around this system. Using LABOCA on APEX we detect strong continuum emission around HD104237-A and system component HD104237-E. Our ASTE spectrum detects a double-peaked ${ }^{12} \mathrm{CO}(3-2)$ line profile towards the system, typical of a rotating disk. The new data are used as constraints with MCFOST to produce a disk model that fits the entire SED as well as the observed CO line profile.
\end{abstract}

\section{Introduction}

DX Cha (HD 104237) is the optically brightest Herbig Ae star (A4-A8Ve) in the sky. At 115 pc, it is part of the young $\epsilon$ Cha stellar group (3-5 Myr; Feigelson et al. 2003). At least five stars are found within 15 arcseconds, and of these two are lower-mass stars that have IR excesses and emission lines, associated to T Tauri type stars (Grady et al. 2004). The brightest member, DX Cha (or HD 104237A), is actually a binary system, where the secondary is a K3 star (HD104237b) at 0.2 AU separation (Böhm et al. 2004). Recent modeling of the optical to mid-infrared SED by Fang et al. (2013) supports the earlier Meeus et al. (2001) interpretation of HD104237's SED as a star being surrounded by a self-shadowed (i.e., non-flared) dust disk. Here we present submillimeter data of the dust and gas disk surrounding DX Cha together with radiative transfer modeling efforts to fit the data.

\section{LABOCA Imaging}

The LABOCA 870 microns continuum map of HD 104237 (Figure 1) shows a clear detection of the primary disk, but also significant extension to the southeast that is consistent with emission from a second disk around the D or E components in the system. Based on the mid-IR detection of a disk around the E component by Grady et al. (2004), we have extracted fluxes using a model of two point sources separated by the 14.9" A-E separation vector (Figure 1). From this method we obtain $870 \mu \mathrm{m}$ fluxes of $154 \mathrm{mJy}$ and $91 \mathrm{mJy}$ for the A and E components. Due to the system configuration, any flux from the 

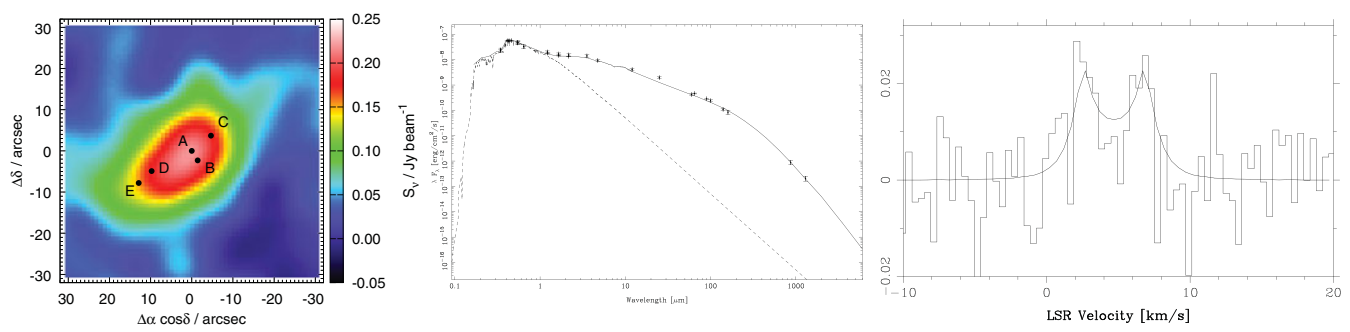

Figure 1. Left: LABOCA map of HD 104237 smoothed with half-beam Gaussian $\left(\mathrm{FWHM}=9.3^{\prime \prime}\right)$, with stellar component positions plotted. Middle: Observed SED of HD 104237 (black points with error bars) plotted against the MCFOST disk model that fits the optical to submillimeter data (solid line). Right: MCFOST CO model of HD 104237 (solid line) fits well the ASTE line, data. Units are in antenna temperatures $\left(\mathrm{T}_{A}^{*}\right)$.

Table 1. HD104237 disk model

\begin{tabular}{lcl}
\hline Physical parameter & Value & Reference \\
\hline Disk total mass: $\left(\mathrm{M}_{\odot}\right)$ & $100 \times \mathrm{M}_{d}$ & \\
Disk dust mass $\mathrm{M}_{d}\left(\mathrm{M}_{\odot}\right)$ & $4 \times 10^{-4}$ & \\
Inner Rim: $\mathrm{R}_{\text {in }}(\mathrm{AU})$ & 0.45 & Tatulli et al. $(2007)$ \\
Characteristic radius $\mathrm{R}_{C}(\mathrm{AU})$ & 90 & \\
Characteristic height $\mathrm{h}_{C}(\mathrm{AU})$ & 6 & \\
Surface density exponent: $\gamma$ & 0.8 & \\
Flaring exponent: $\psi$ & 0.0 & \\
Inclination angle: $\mathrm{i}(\mathrm{deg})$ & 31 & \\
Systemic Velocity: $\mathrm{v}_{\mathrm{lsr}}(\mathrm{km} / \mathrm{s})$ & 4.2 & \\
\hline
\end{tabular}

$\mathrm{D}$ component will mostly be incorporated in the $\mathrm{E}$ component flux, and any emission from the $\mathrm{B}$ and $\mathrm{C}$ components will be included in the $\mathrm{A}$ component flux.

\section{Results and Conclusions}

The 3D radiative transfer code MCFOST (Pinte et al. 2006) was used to model the SED and the molecular emission of HD104237A, assuming that both the infrared excess and ${ }^{12} \mathrm{CO}(3-2)$ emission stem from a circumstellar disk (Figure 1). We find the SED and $\mathrm{CO}$ line can be well fit by a non-flared disk $90 \mathrm{AU}$ in radius and of $4 \times 10^{-2} \mathrm{M}_{\odot}$ total mass. The parameters of this representative model are shown in Table 1 . The CO surface brightness distributions predicted by the standard model over predicts the disk intensity by factors of 2-3 (assuming $\mathrm{CO} / \mathrm{H}_{2}$ ratios of $10^{-4}$ ). By also incorporating the freeze-out of ${ }^{12} \mathrm{CO}$ molecules into dust grains at $\mathrm{T}<20 \mathrm{~K}$, however, the model fits well the line data. HD 104237 is a unique laboratory to study the extreme of SED-predicted flat disks. Resolved submillimeter images with existing interferometers (e.g. ALMA) are required to further constrain the physical and chemical structures of the disk.

\section{References}

Böhm, T., Catala, C., Balona, L., \& Carter, B. 2004, A\& A, 427, 907

Fang, M., van Boekel, R., Bouwman, J., et al. 2013, A\&SA, 549, A15

Feigelson, E. D., Lawson, W. A., \& Garmire, G. P. 2003, ApJ, 599, 1207

Grady, C. A., Woodgate, B., Torres, C. A. O., et al. 2004, ApJ, 608, 809

Meeus, G., Waters, L. B. F. M., Bouwman, J., et al. 2001, A\&A, 365, 476

Pinte, C., Ménard, F., Duchêne, G., \& Bastien, P. 2006, A\&SA, 459, 797

Tatulli, E., Isella, A., Natta, A., et al. 2007, A\&A, 464, 55 